\title{
Disentangling stellar activity from exoplanetary signals with interferometry
}

\author{
Roxanne Ligi $^{1}$, a, Denis Mourard ${ }^{1}$, Anne-Marie Lagrange $^{2}$, Karine Perraut $^{2}$, and Andrea Chiavassa ${ }^{1}$ \\ 1 Laboratoire Lagrange, UMR 7293 UNS-CNRS-OCA, Boulevard de l'Observatoire, CS 34229, 06304 \\ NICE Cedex 4, France \\ 2 UJF-Grenoble1/CNRS-INSU, Institut de Planétologie et d'Astrophysique de Grenoble, UMR 5274, \\ Grenoble, F-38041, France
}

\begin{abstract}
Stellar activity can express as many forms at stellar surfaces: dark spots, convective cells, bright plages. Particularly, dark spots and bright plages add noise on photometric data or radial velocity measurements used to detect exoplanets, and thus lead to false detection or disrupt their derived parameters. Since interferometry provides a very high angular resolution, it may constitute an interesting solution to distinguish the signal of a transiting exoplanet and that of stellar activity. It has also been shown that granulation adds bias in visibility and closure phase measurements, affecting in turn the derived stellar parameters. We analyze the noises generated by dark spots on interferometric observables and compare them to exoplanet signals. We investigate the current interferometric instruments able to measure and disentangle these signals, and show that there is a lack in spatial resolution. We thus give a prospective of the improvements to be brought on future interferometers, which would also significantly extend the number of available targets.
\end{abstract}

\section{Introduction}

The discovery of the first exoplanet around a solar-type star [1] has opened up the wide field of exoplanet detection and characterization. The two most prolific methods used to detect exoplanets are the radial velocity (RV) measurements and the transit method. While the first one provides the ratio of the minimum mass of the exoplanet and the stellar mass, $M_{\mathrm{p}} \sin (i) / M_{\star}$, the second one gives the ratio of their radii, $R_{\mathrm{p}} / R_{\star}$. These ratios are much amore accurate than the stellar parameters, $M_{\star}$ and $R_{\star}$, which constitutes a limitation for exoplanet characterization. The determination of exoplanet density, composition, and habitability is only possible if exoplanet parameters are known with a sufficient accuracy (for example, we can estimate that $2 \%$ accuracy on $R_{\mathrm{p}}$ is needed because it is required in some models; see, e.g., [2]). It is therefore essential to improve stellar parameters accuracy to achieve this goal. Meanwhile, stellar activity, that expresses under different forms (magnetic spots, granulation, bright plages), constitutes another limitation in exoplanet detection and characterization. It adds noise in RV measurements (see, e.g., [3]; [4]; [5]; [6]) and transit light-curves, and also on interferometric observables [7], and should thus be analyzed and quantified for a better estimate of exoplanet parameters. We first give, in Sect. 2, a few solutions to obtain accurate stellar parameters, particularly with interferometry, and the limitations of this method. In Sect. 3, we show how interferometry could be a good solution to characterize exoplanets and distinguish it from stellar activity signals.

\footnotetext{
a e-mail: roxanne.ligi@oca.eu
}

This is an Open Access article distributed under the terms of the Creative Commons Attribution License 4.0, which permits unrestricted use, distribution, and reproduction in any medium, provided the original work is properly cited. 


\section{Stellar parameters : determination and limitations}

Several methods are used to estimate stellar parameters. For instance, asteroseismology allows measuring stellar oscillation frequencies, which gives access to stellar gravity, radius, and density through models. In particular, the stellar radius is very important both for stellar and planetary characterization, as shown in the Introduction. A more direct method to derive the stellar radius is to use interferometry, that allows measuring complex visibilities. The visibility function represents the Fourier transform of the brightness distribution of the source; this complex function is sampled by interferometric observables and can be used either for recovering images of the source or to adjust model parameters to the measurements. It is decomposed into two parts: usually, we use the squared of the amplitude called the squared visibility, and its argument, called the phase $\phi$, described as $\phi=\tan ^{-1}(\mathfrak{I}(V))$, where $\mathfrak{R}$ and $\mathfrak{J}$ define the real and imaginary part of the complex number $V$.

In the case of stars, measuring the squared visibility allows estimating their angular diameter with almost model-independent method (see, e.g., [8]). It is then used in stellar models to derive the other parameters: effective temperature, radius, mass, age.

However, current interferometers are limited to the observation of bright objects, which generally do not include transiting exoplanet host stars (see Fig. 7 in [9]). This is mainly due to insturmental bias: CoRoT and Kepler were the two most important providers of exoplanets but only focused on faint stars. In general, interferometers measure visibilities in the first lobe only, or are not able to measure closure phase (CP), making the measurement of limb darkening and smaller effects, such as stellar activity effects and transiting exoplanet signals, impossible. However, due to its high angular resolution, this method could constitute a good solution to characterize exoplanets.

\section{The charactarization of exoplanets and stellar activity}

The code COMETS (code for modeling exoplanets and spots, [9]) allows measuring the complex visibility of a star with a transiting exoplanet, a dark magnetic spot, or both, and thus characterizing their signals on interferometric observables. The detailed methodology and calculations are presented in [9].

\subsection{Minimum Baseline Length}

We first estimate the Minimum Baseline Length (MBL) needed to detect a given signal caused by a transiting exoplanet or a spot in the visible wavelength $(720 \mathrm{~nm})$ considering several parameters and a host star of 1 mas. To that purpose, we either consider the absolute signal, that is $\left|V_{\mathrm{p}+\star}^{2}-V_{\star}^{2}\right|>S$, where $V_{\star}$ and $V_{\mathrm{p}+\star}$ are the visibility modulus of the star alone and of the star with the transiting exoplanet. We calculate the MBL for signals $\mathrm{S}$ of $0.5 \%, 1.0 \%$, and $2.0 \%$ in squared visibilities, and $2^{\circ}$ and $20^{\circ}$ in phases. This methods implies that the signal is limited by a fixed noise, which is very conservative. Another way of calculating the MBL is to consider that the signal-to-noise ratio $(\mathrm{S} / \mathrm{N})$ is photon-noise limited. This means that we calculate the MBL for a signal such as $\frac{\left|V_{\mathrm{p}}^{2}-V_{\star}^{2}\right|}{V_{\star}^{2}}>\frac{1}{S / N}$. A $\mathrm{S} / \mathrm{N}$ of 5 corresponds to bad observing conditions and a $\mathrm{S} / \mathrm{N}$ of 20 to good ones. The most important results are given in Table 1.

The longest baselines in the world currently reach $330 \mathrm{~m}$ and are located on the CHARA array [10]. According to this model, this is sufficient to detect a large transiting exoplanet $\left(\theta_{\mathrm{p}}=0.09\right.$ mas $)$ or a dark spot (that is with a low temperature, $T_{\mathrm{s}}=4875 \mathrm{~K}$ ) considering absolute variations. Considering the $\mathrm{S} / \mathrm{N}$ leads to the detection of smaller exoplanets and brighter spots. However, these signals are weak, and detecting an exoplanet or a spot by measuring the phases would be more realistic and feasible as the signals they cause are detectable.

In the case of a transiting exoplanet and absolute variations, the MBL can be written as an empirical formulae derived from these results. Using a least-squares method, we find that the best fit is 


\begin{tabular}{c|ccccc|cc}
\hline \hline & \multicolumn{5}{|c|}{ Visibility } & \multicolumn{2}{c}{ Phases } \\
& $0.5 \%$ & $1 \%$ & $2 \%$ & $\mathrm{~S} / \mathrm{N}=5$ & $\mathrm{~S} / \mathrm{N}=20$ & $2^{\circ}$ & $20^{\circ}$ \\
\hline$\theta_{\star}$ & $0.3 \mathrm{mas}$ & $0.3 \mathrm{mas}$ & $/$ & $0.3 \mathrm{mas}$ & $0.3 \mathrm{mas}$ & $0.3 \mathrm{mas}$ & $0.3 \mathrm{mas}$ \\
& $160 \mathrm{~m}$ & $250 \mathrm{~m}$ & & $515 \mathrm{~m}$ & $364 \mathrm{~m}$ & $340 \mathrm{~m}$ & $600 \mathrm{~m}$ \\
\hline$\theta_{\mathrm{p}}$ & $0.09 \mathrm{mas}$ & $0.14 \mathrm{mas}$ & $0.20 \mathrm{mas}$ & $0.04 \mathrm{mas}$ & $0.04 \mathrm{mas}$ & $0.04 \mathrm{mas}$ & $0.04 \mathrm{mas}$ \\
& $110 \mathrm{~m}$ & $110 \mathrm{~m}$ & $90 \mathrm{~m}$ & $190 \mathrm{~m}$ & $180 \mathrm{~m}$ & $180 \mathrm{~m}$ & $190 \mathrm{~m}$ \\
\hline$T_{\mathrm{s}}$ & $4870 \mathrm{~K}$ & $4030 \mathrm{~K}$ & $/$ & $5170 \mathrm{~K}$ & $5170 \mathrm{~K}$ & $5170 \mathrm{~K}$ & $5170 \mathrm{~K}$ \\
& $100 \mathrm{~m}$ & $108 \mathrm{~m}$ & & $175 \mathrm{~m}$ & $158 \mathrm{~m}$ & $184 \mathrm{~m}$ & $192 \mathrm{~m}$ \\
\hline$\alpha_{\mathrm{p}}$ & $\mathrm{C}$ & $\mathrm{C}$ & $\mathrm{C}$ & $\mathrm{C}$ & $\mathrm{C}$ & $0.3 \mathrm{mas}$ & $0.3 \mathrm{mas}$ \\
& $46 \mathrm{~m}$ & $73 \mathrm{~m}$ & $250 \mathrm{~m}$ & 155 & $110 \mathrm{~m}$ & $90 \mathrm{~m}$ & $180 \mathrm{~m}$ \\
\hline
\end{tabular}

Table 1: Summary of the results obtained for the MBL tests. For each tested parameter (stellar diameter $\theta_{\star}$, exoplanet diameter $\theta_{\mathrm{p}}$, spot temperature $T_{\mathrm{s}}$, and exoplanet position on the stellar disc $\alpha_{\mathrm{p}}$ ) we list the lowest or highest value of our test for which a MBL is found and the value of the corresponding MBL (see text). C stands for constant.

$$
M B L=\left(17 \times S^{0.1}\right) \times\left(\frac{\theta_{\mathrm{p}}}{\theta_{\star}}-\sqrt{\frac{S}{2}}\right)^{-1.7 \times S^{0.2}} \times\left(\frac{\theta_{\star}}{1 \mathrm{mas}}\right)^{-1} \times \frac{\lambda}{720.10^{-9}} \mathrm{~m},
$$

where $S$ represents the signal to be detected ans $\lambda$ the observing wavelength.

\subsection{Detectability}

Having long enough baselines to detect a signal does not mean that an intrument can actually do it: it depends on its sensitivity. Therefore, we have to consider the spatial frequency at which the signal caused by the exoplanet or the spot can be detected. Using COMETS, we computed the signal caused by an exoplanet as a function of its diameter for several spatial frequencies; in the first visibility lobe, close to the first null, and in the second lobe.

In the first visibility lobe, the signal caused by the exoplanet is weak. A 0.10 mas exoplanet causes the highest signal of $0.05 \%$, while only a large exoplanet ( $\sim 0.20$ mas) can be detected with a $\mathrm{S} / \mathrm{N}$ of 20. These results are similar considering the phase: under a diameter of 0.20 mas, the signal caused by an exoplanet is below $2^{\circ}$.

Small exoplanets start to be detectable close to the first null and in the second lobe. The phase seems to be more adapted to detect them: the phase signal reaches $2^{\circ}$ for a $\sim 0.09$ mas exoplanet in the first visibility null, and for a $\sim 0.06$ mas exoplanet in the second lobe. It seems possible to detect similar exoplanets in visibility measurements; for example, under good observing conditions $(\mathrm{S} / \mathrm{N}=20)$, an exoplanet of $\sim 0.06$ mas could be detectable. However, condidering the absolute signal, only exoplanets larger than $\sim 0.13$ mas cause a signal of at least $0.05 \%$.

Detecting an Earth-sized or a Neptune-sized planet is thus very difficult because instruments do not reach the needed accuracy on measurements. It should not be forgotten that phase and CP measurements depend at first on the ability to measure the visibility because they are higher order measurements; having a phase measurement means that the visibility measurement is not too weak. Most of instruments able to measure $\mathrm{CP}$ can only do it in the first lobe, which is not enough to detect transiting exoplanets.

\subsection{Distinguishing exoplanet and spot}

The other limitation in an exoplanet characterization, after the actual possibility of detecting it, is to distinguish its signal from a dark spot signal. Figure 1 shows the difference between the visibility (upper planels) of a single star of 1 mas and a star with a transiting exoplanet of diameter 0.01 mas 

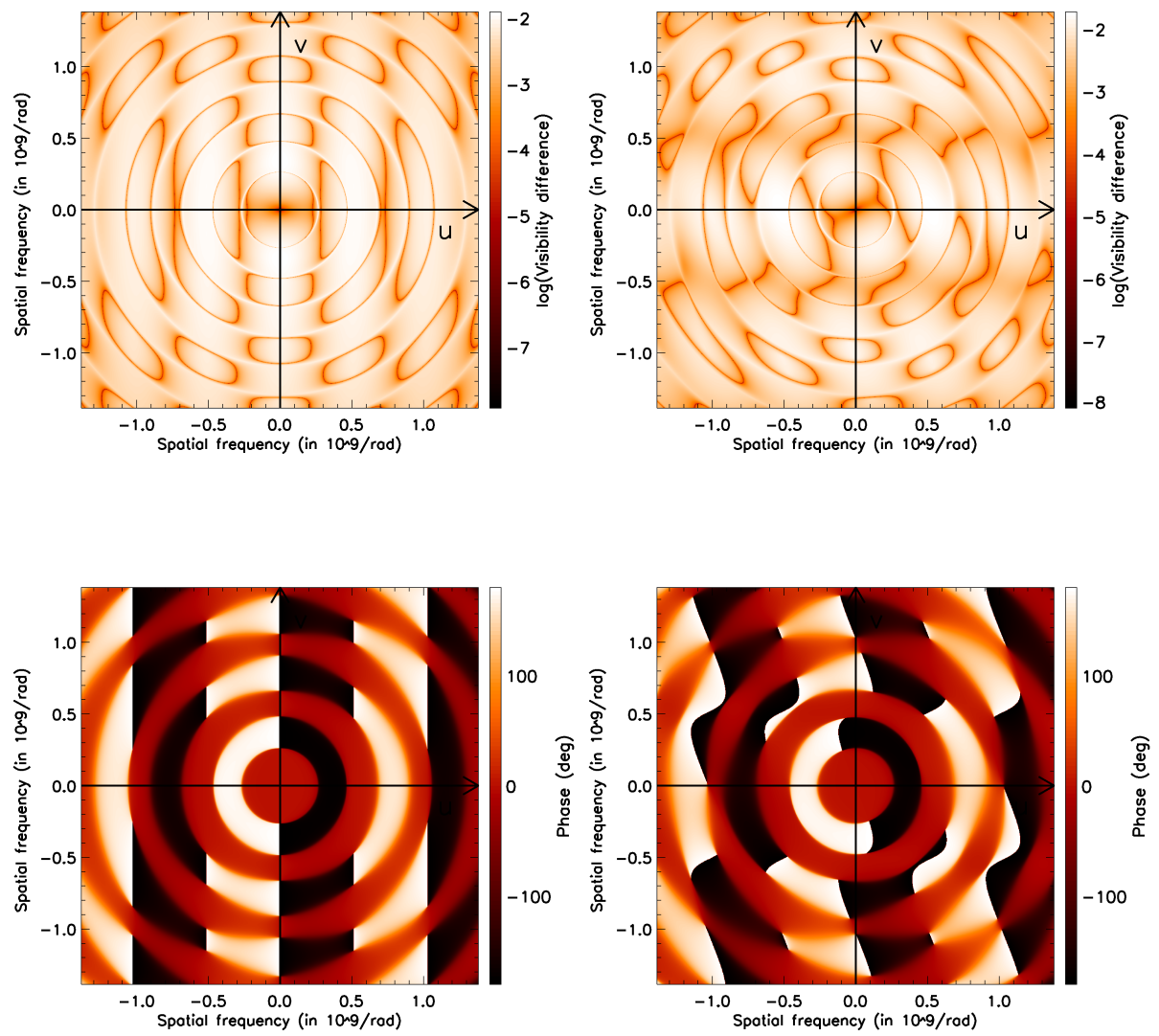

Fig. 1: Map of the signal induced by an exoplanet at $(0.2,0.0)$ mas and a spot at $(0.2,0.2)$ mas on the stellar disc in the visibility modulus (log scale, upper panels) and the phases (bottom panels), for a 1 mas star. Left: exoplanet alone with $\theta_{\mathrm{p}}=0.10$ mas. Middle: exoplanet of diameter $\theta_{\mathrm{p}}=0.10$ mas with a spot of diameter $\theta_{\mathrm{s}}=0.10$ mas.

(left), and the corresponding phases (bottom panels). The right panels show this same system with an additional dark spot of diameter 0.01 mas.

The star with an exoplanet and/or a spot has a different signal from that of a single star, whose phase is $0 \pm 180^{\circ}$. The signal of the exoplanet and that of the spot are mixed up until the second lobe of visibility. If the exoplanet is smaller than the spot $\left(\theta_{\mathrm{p}}=0.05\right.$ mas), the signal in the phase is totally disturbed by the spot. Measurements before and after the transit are thus essential to distinguish the exoplanet signal from that of the spot. It is also important because if interferometers accuracy reaches the signal caused by an exoplanet or a spot, stellar parameters derived from these measurements can be modified. For example, they can either lead to a bad estimation of exoplanetary or stellar angular diameter. This last parameters is generally measured in the first visibility lobe: at higher spatial frequency, the signal is sensitive to thinner effects, such as stellar activity ones. 


\section{Conclusion}

COMETS allows computing the signal caused by a transiting exoplanet and/or a spot on interferometric observables. It has been used to calculate the baseline lengths needed to detect an exoplanet or a spot, the ability of interferometers to detect such signals in terms of accuracy, and the noise generated by darks spots on exoplanet signals. We have shown that the main limitation to detect exoplanets lies in the accuracy of instruments, and that spot can mimic exoplanet signals. Distinguishing between both signals requires several observations and a significant improvement in accuracy. This is also valid to characterise Earth-sized and Neptune-sized exoplanets. Finally, the visible domain provides a better angular resolution than the infrared domain at similar baseline, and therefore appear more suitable. However, no current interferometer is able to characterize exoplanets: the visible instruments on the CHARA array, VEGA ([11]; [12]) and PAVO [13], are not accurate enough, and baselines at the VLTI are too short. Most of exoplanets detected until now are too faint to be observable with current interferometers. In the next years, new spatial missions, such as PLATO [14], TESS [16], or CHEOPS [15], will allow detecting exoplanets around bright stars, and complementary observations from the ground, especially by interferometers, will become possible.

\section{References}

1. Mayor, M. \& Queloz, D. 1995, Nature, 378, 355

2. Valencia, D., et al. 2013, ApJ, 775, 10

3. Lagrange, A.M., et al. 2010, A\&A, 512, A38

4. Meunier, N., et al. 2010, A\&A, 512, A39

5. Boisse, I., et al. 2011, A\&A, 528, A4

6. Dumusque, X., et al. 2011, A\&A, 527, A82

7. Chiavassa, A., et al. 2014, A\&A, 567, A115

8. Ligi, R., et al. 2012, A\&A, 545, A5

9. Ligi, R., et al. 2014, A\&A, in press, 2014arXiv1410.5333L

10. McAlister, H. A., et al. 2012, in Society of Photo-Optical Instrumentation Engineers (SPIE) Conference Series, Vol. 8445

11. Mourard, D., et al. 2009, A\&A, 508, 1073

12. Ligi, R., et al. 2013, Journal of Astronomical Instrumentation, 02, 1340003

13. Ireland, M. J., et al. 2008, in Society of Photo-Optical Instrumentation Engineers (SPIE) Conference Series, Vol. 7013

14. Rauer, H. \& Catala, C. 2012, in EGU General Assembly Conference Abstracts, Vol. 14, 7033

15. Broeg, A., et al. 2013, in European Physical Journal Web of Conferences, Vol. 47, 3005

16. Ricker, G. R., et al. 2010, in Bulletin of the American Astronomical Society, Vol. 42, \#215, 450.06 